\title{
POSSIBILIDADES E LIMITAÇÕES NO USO DA TEMPERATURA EM CROMATOGRAFIA LÍQUIDA DE FASE REVERSA
}

Endler Marcel Borges, Carla B. G. Bottoli e Carol.H. Collins*

Instituto de Química, Universidade Estadual de Campinas, CP 6154, 13083-970 Campinas - SP, Brasil

Recebido em 16/9/09; aceito em 30/11/09; publicado na web em 8/4/10

\begin{abstract}
POSSIBILITIES AND LIMITATIONS OF USING TEMPERATURE IN REVERSED PHASE LIQUID CHROMATOGRAPHY. High-temperature liquid chromatography (HTLC) is a technique that presents a series of advantages in liquid phase separations, such as: reduced analysis time, reduced pressure drop, reduced asymmetry factors, modified retentions, controlled selectivities, better efficiencies and improved detectivities, as well as permitting green chromatography. The practical limitations that relate to instrumentation and to stationary phase instability are being resolved and this technique is now ready to be applied for routine determinations.
\end{abstract}

Keywords: high temperature liquid chromatography; stationary phase stability; green chromatography.

\section{INTRODUÇÃO}

O termo alta temperatura em cromatografia líquida (High Temperature Liquid Chromatography, HTLC) se refere ao uso de temperaturas entre 40 a $200{ }^{\circ} \mathrm{C}$. O uso da temperatura como variável em cromatografia líquida é recente, embora Antia e Horvath ${ }^{1}$ tenham descrito seu uso em 1988. A principal vantagem desta técnica é a redução no tempo de análise, característica que é de grande interesse prático para os usuários de cromatografia. Mas até o momento, esta técnica reside quase exclusivamente no meio acadêmico por causa de algumas limitações, como a falta de instrumentação específica para tal fim e a instabilidade das fases estacionárias. Desta forma, esta revisão se dedica à discussão das vantagens e limitações desta técnica, bem como os avanços recentes quanto à instrumentação e fases estacionárias, os quais devem impulsionar a migração desta técnica para o meio industrial.

\section{ASPECTOS TEÓRICOS}

\section{Influência da temperatura sobre a pressão}

A pressão gerada pela passagem de fase móvel (FM) por uma coluna pode ser descrita pela equação de Kozeny-Carman:2,3 $\Delta \mathrm{P}$ $=(\eta \mathrm{FL}) /\left(\mathrm{K}_{0} \pi \mathrm{r}^{2} \mathrm{dp} \mathrm{p}^{2}\right)$, onde $\mathrm{K}_{0}$ é a permeabilidade específica, $\eta$ é a viscosidade da FM, F é a vazão da FM, r é o raio interno da coluna e dp é o diâmetro médio das partículas da fase estacionária (FE) que recheiam a coluna. A viscosidade decresce exponencialmente em função da temperatura, ${ }^{2,3}$ assim, o aumento da temperatura permite: a utilização de maiores vazões; o emprego de partículas com menores diâmetros; a utilização de colunas com maior comprimento ou conectadas em série.

Um exemplo de como o aumento da temperatura permite o uso de maiores vazões é o trabalho de Yang et $a .^{2}$ onde uma coluna ZirChrom-PS (50 x 4,6 mm, dp 2,5 $\mu \mathrm{m}$ ) utilizada a $25^{\circ} \mathrm{C}$ e com FM acetonitrila:água, 25:75 v/v, a uma vazão de $2 \mathrm{~mL} / \mathrm{min}$ gera uma pressão de 400 bar, alcançando o limite máximo de pressão de um sistema convencional de cromatografia líquida de alta eficiência (High Performance Liquid Chromatography, HPLC). Quando a temperatura é elevada a $150{ }^{\circ} \mathrm{C}$, a pressão é reduzida a 60 bar, permitindo o uso de uma vazão de $14 \mathrm{~mL} / \mathrm{min}$ sem exceder o limite de pressão da bomba.

*e-mail: chc@iqm.unicamp.br
As micropartículas $(\mathrm{dp}<2 \mu \mathrm{m})$ têm pequena resistência à transferência de massa a altas vazões. Por isso podem ser utilizadas com pouca penalidade quanto à perda de eficiência. Neste caso a vazão é limitada pelo limite de pressão da bomba, uma vez que a pressão aumenta ao quadrado em função da redução de dp. Petersson e Euerby ${ }^{4}$ estudaram várias micropartículas para cromatografia líquida de ultra pressão (Ultra High Pressure Liquid Chromatography, UHPLC) e constataram que, quando a temperatura é aumentada de 20 a $90{ }^{\circ} \mathrm{C}$ em FM acetonitrila:água, 20:80 v/v, a uma vazão constante de 0,6 $\mathrm{mL} / \mathrm{min}$, a pressão do sistema é reduzida em $60 \%$, permitindo que colunas produzidas para UHPLC $(\mathrm{dp}<2 \mu \mathrm{m})$ possam ser utilizadas num sistema convencional de HPLC (pressão máxima de 400 bar).

$\mathrm{O}$ aumento da temperatura permite o uso de colunas com maiores comprimentos ou acopladas em série, como demonstrado por Sandra e Vanhoenacker ${ }^{5}$ onde uma temperatura de $60^{\circ} \mathrm{C}$ permitiu que oito colunas Zorbax SB300-C18 (25 x $2.1 \mathrm{~mm}$, dp $5 \mu \mathrm{m})$ fossem conectadas em série e utilizadas a uma vazão de $0,2 \mathrm{~mL} / \mathrm{min}$ em FM acetonitrila:água, 40:60 v/v, fornecendo eficiência de 200000 pratos a uma pressão de apenas 425 bar. Edge et al. ${ }^{6}$ operando uma coluna $(100$ x 2,1 mm, dp $1,7 \mu \mathrm{m})$ a $60{ }^{\circ} \mathrm{C}$ em FM 0,1\% ácido fórmico a uma vazão de $0,4 \mathrm{~mL} /$ min, atingiram o limite máximo de pressão de uma bomba de UHPLC (1.000 bar). Aumentando a temperatura para $180{ }^{\circ} \mathrm{C}$, foi possível conectar três colunas em série e aumentar a vazão para $1 \mathrm{~mL} / \mathrm{min}$, mantendo a mesma pressão. Outros exemplos da combinação de altas temperaturas com a técnica de UHPLC encontram-se na literatura. ${ }^{7-12}$

\section{Influência da temperatura sobre o fator de retenção}

O fator de retenção, $\mathrm{k}$, de um soluto é determinado pela razão das quantidades das suas moléculas que ficam retidas na fase estacionária, $\mathrm{n}_{\mathrm{S}}$, ou percorrendo a coluna na fase móvel, $\mathrm{n}_{\mathrm{M}}$, e também é relacionado à razão dos tempos que as moléculas ficam na fase estacionária, $t_{R}$, e na fase móvel, $t_{M}\left(k=n_{s} / n_{M}=\left(t_{R}-t_{M}\right) / t_{M}\right)$. A variação do fator de retenção de um soluto em função da temperatura é descrita pela equação de Van Hoff, ${ }^{3} \ln (\mathrm{k})=-\Delta \mathrm{H}^{\mathrm{o}} / \mathrm{RT}+\Delta \mathrm{S}^{\circ} / \mathrm{R}+\ln (\beta)$, onde $\Delta \mathrm{H}^{\circ}$ e $\Delta S^{\circ}$ são as entalpias e entropias de retenção, T é a temperatura em $\mathrm{K}$, R é a constante dos gases $\left(8,314 \mathrm{~J} \mathrm{~K}^{-1} \mathrm{~mol}^{-1}\right)$ e $\beta$ é a razão de fases. Solutos pequenos e neutros possuem pequenas entalpias de retenção e apresentam variações pequenas e lineares em seus tempos de retenção em função da temperatura, enquanto solutos como proteínas, ${ }^{13}$ polipeptídios $^{14,15}$ e bases ${ }^{16,17}$ apresentam variações pronunciadas e não lineares em seus tempos de retenção em função da temperatura. 
Szabelski et al. ${ }^{13}$ estudaram a influência da temperatura sobre uma série de proteínas empregando uma coluna Symmetry C8 (150 x 3.9 $\mathrm{mm}$, dp $5 \mu \mathrm{m}$ ) e observaram que o fator de retenção destas proteínas variou em até 10 vezes na faixa de temperatura de 20 a $50{ }^{\circ} \mathrm{C}$.

Como o pH da FM e o pKa de solutos ionizáveis variam em função da temperatura, ${ }^{16-24}$ pequenas variações na temperatura podem alterar de maneira acentuada os fatores de retenção destes solutos. Buckenmaier et al. ${ }^{23}$ e Cheng et al. ${ }^{24}$ observaram variações entre 40 e $70 \%$ nos fatores de retenção de antidepressivos tricíclicos variando a temperatura de 30 a $60{ }^{\circ} \mathrm{C}$ em colunas de nova geração com poucos silanóis residuais (XTerra- $\mathrm{RP}_{18}$ e XTerra MS $\mathrm{C}_{18}$ ).

Heinisch et al..$^{16,17}$ estudaram o comportamento cromatográfico de solutos farmacêuticos básicos em uma faixa de temperatura de 30 a $170{ }^{\circ} \mathrm{C}$ e observaram que os fatores de retenção variaram de forma sigmoide em função da temperatura nas colunas Nucleodur Gravity C18 (sílica), Hypercarb (carbono grafitizado) e PLRP-S (polímero de poliestireno-divinilbenzeno). A variação dos fatores de retenção dos solutos básicos foi diferente em FM acetonitrila: $15 \mathrm{mmol} / \mathrm{L}$ fosfato de potássio a $\mathrm{pH}$ 6, 30:70 v/v, da variação observada com tampão acetato de amônio na mesma coluna, Nucleodur Gravity $\mathrm{C} 18,{ }^{16}$ demonstrando que a variação no fator de retenção de solutos ionizáveis em função da temperatura depende fortemente do tipo de tampão utilizado. Gagliardi et al. ${ }^{21}$ demonstraram como uma variação de temperatura de 20 a $60{ }^{\circ} \mathrm{C}$ e o tipo de tampão utilizado afetam a retenção de solutos ionizáveis. O fator de retenção do soluto ácido fenbufeno é reduzido de 16,6 a 7,9 quando a temperatura é aumentada de 20 a $60{ }^{\circ} \mathrm{C}$ em FM acetonitrila: piperazina a $\mathrm{pH} 4,9,30: 70 \mathrm{v} / \mathrm{v}$, enquanto com tampão acetato, a mesma variação na temperatura resulta em uma pequena redução na retenção, 15,9 a 14,4. O fator de retenção do soluto básico cinchonina é reduzido de 12,8 a 5,7 em tampão Tris a pH 8,5 com o aumento da temperatura, mas em tampão fosfato o aumento da temperatura resulta em um ligeiro aumento da retenção, 6,0 a 6,5.

Mesmo solutos pequenos e apolares como alquilbenzenos não apresentam comportamento linear em faixas de temperatura de 30 a $200{ }^{\circ} \mathrm{C} .{ }^{25-28}$ Liu et al.${ }^{26}$ relataram entalpia de retenção para o tolueno de $-4,2 \mathrm{kcal} / \mathrm{mol}$ em uma faixa de temperatura de 32 a $97{ }^{\circ} \mathrm{C}$ e de $-8,2$ $\mathrm{kcal} / \mathrm{mol}$ em uma faixa de temperatura de 97 a $200{ }^{\circ} \mathrm{C}$ em FM $100 \%$ água com a coluna XBridge C18. Al-Khateeb e Smith ${ }^{25}$ observaram comportamentos similares nas colunas XBridge Phenyl e XTerra Phenyl. Cabe ressaltar que quanto maiores os solutos maiores as entalpias de retenção e, portanto, maiores as variações da retenção em função da temperatura. Al-Khateeb e Smith ${ }^{29}$ observaram altas entalpias de retenção, -17 a $-67 \mathrm{~kJ} / \mathrm{mol}$, para esteroides em uma coluna XTerra MS C $\mathrm{M}_{18}$ em faixas de temperatura de 50 a $120{ }^{\circ} \mathrm{C}$ com FM nas composições metanol:água de 50:50 a 20:80, v/v. A redução da percentagem de metanol na FM ocasiona um aumento no fator de retenção e, consequentemente, em uma maior entalpia de retenção.

\section{Influência da temperatura sobre a eficiência cromatográfica}

A eficiência em cromatografia é medida pelo numero de pratos (N) ou altura do prato $(\mathrm{H})$, onde $\mathrm{H}=\mathrm{N} / \mathrm{L}$ (L é o comprimento da coluna), e a altura do prato é uma função da velocidade linear (u) expressa pela equação de van Deemter $(\mathrm{H}=\mathrm{A}+\mathrm{B} / \mathrm{u}+\mathrm{Cu})$. O termo A depende dos caminhos múltiplos percorridos pelo soluto, o termo $\mathrm{B}$ depende da difusão longitudinal do soluto, e o termo $\mathrm{C}$ depende da transferência de massa entre a FM e a FE. A velocidade linear em que a altura do prato tem seu valor mínimo é denominada velocidade linear ótima, $\mathrm{u}_{\text {otima: }}$.

As vantagens obtidas com o aumento da temperatura frente à redução do tempo de análise são descritas pelas curvas de van Deemter no exemplo da Figura $1,{ }^{2}$ onde a $25^{\circ} \mathrm{C}$ o uso de velocidades lineares maiores que $\mathrm{u}_{\text {otima }}$ resultam num aumento acentuado da altura de prato. Por sua vez, o aumento da temperatura facilita a transferência de massa entre a FM e a FE, atenuando as curvas de van Deemter. O aumento da temperatura de 25 a $180{ }^{\circ} \mathrm{C}$ resulta em uma redução do termo $\mathrm{C}$, permitindo o uso de velocidades lineares maiores que $\mathrm{u}_{\text {otima }}$ em detrimento de apenas um pequeno aumento na altura de prato. A difusão longitudinal (termo B) aumenta com o aumento da temperatura, fazendo com que $\mathrm{u}_{\text {otima }}$ se desloque para valores mais elevados, uma vez que a baixas velocidades lineares as curvas de van Deemter são dominadas pela difusão longitudinal. ${ }^{2}$

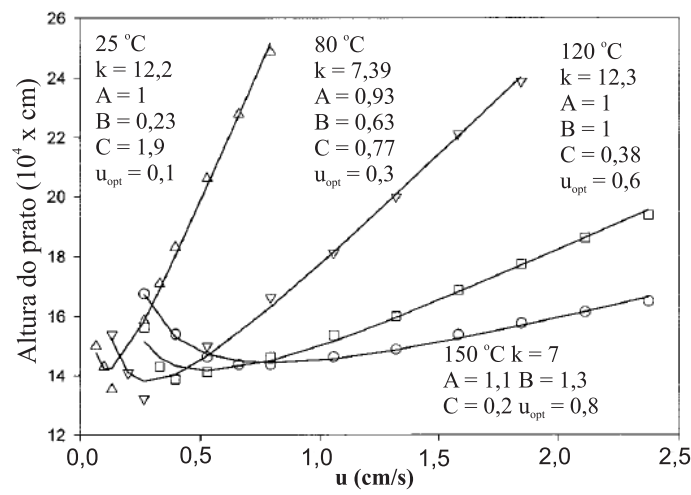

Figura 1. Altura do prato vs velocidade linear (u). Coluna: ZirChrom-PS (50x 4,6 mm, dp 2,5 $\mu \mathrm{m})$. Para $25^{\circ} \mathrm{C}$ : soluto decanofenona e FM acetonitrila:água, 40:60 v/v. Para $80^{\circ} \mathrm{C}$ : soluto dodecanofenona e FM acetonitrila:água, 40:60 v/v. Para $120^{\circ} \mathrm{C}$ : soluto dodecanofenona.e FM acetonitrila:água, 30:70 v/v. Para $150{ }^{\circ} \mathrm{C}$ : soluto tetradecanofenona e FM acetonitrila:água 25:75 v/v. Adaptado da ref. 2

\section{Influência da temperatura sobre a assimetria}

A assimetria dos picos cromatográficos é um problema frequente em cromatografia quando os solutos estudados possuem funções ionizáveis, os quais podem ter interações secundárias com os silanóis residuais da sílica da FE, resultando em dois mecanismos distintos de retenção, com cinéticas diferentes de transferência de massa. Quanto maior forem as diferenças nas cinéticas de transferência de massa maior será a assimetria. ${ }^{30}$

A cinética de transferência de massa aumenta com a temperatura, reduzindo a assimetria, como ilustrado na Figura 2, a qual evidencia que uma pequena variação da temperatura $\left(30\right.$ a $\left.60{ }^{\circ} \mathrm{C}\right)$ resulta em uma redução acentuada da assimetria dos dois solutos altamente básicos em $\mathrm{pH} 7$, valor de $\mathrm{pH}$ no qual as interações secundárias são mais pronunciadas. ${ }^{31}$ Por outro lado, em $\mathrm{pH} 3$, onde as interações secundárias são menos pronunciadas (supõe-se que a maioria dos silanóis residuais estejam protonados) não são observadas melhorias no fator de assimetria com o aumento da temperatura. ${ }^{31}$

Em estudo posterior ${ }^{23} \mathrm{com}$ as colunas Inertisil ODS-3V (250 x 4,6 mm, dp $5 \mu \mathrm{m})$ e XTerra RP18 (150 x 4,6 mm, dp $5 \mu \mathrm{m})$ em FM acetonitrila: tampão fosfato a pH 7,8, 60:40 v/v, foi observado que o aumento da temperatura de 30 a $60{ }^{\circ} \mathrm{C}$ melhorou de maneira acentuada o fator de assimetria de bases fortes como amitriptilina, nortriptilina, protriptilina e quinina na coluna Inertisil ODS-3V, enquanto uma melhora discreta foi observada na coluna XTerra RP18, provavelmente devido à menor quantidade de silanóis residuais nesta coluna de nova geração.

Normalmente a assimetria é observada apenas para solutos ionizáveis quando se utiliza FE à base de sílica e a observação de assimetria para solutos neutros se deve ao mau enchimento da coluna. No entanto, a assimetria é frequentemente observada com solutos neutros em FE à base de óxido de zircônio, polímeros ou carbono 


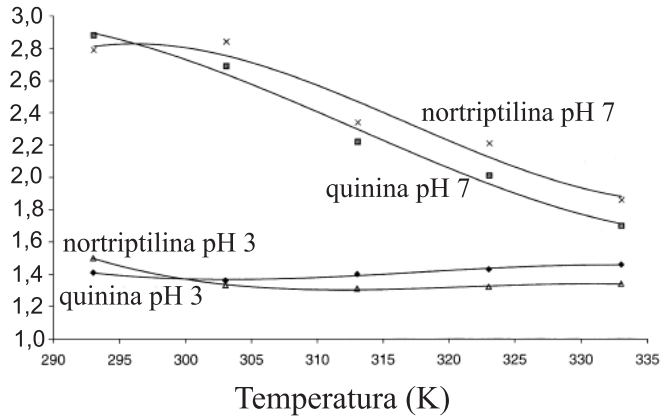

Figura 2. Variação dos fatores de assimetria em função da temperatura. Coluna: Inertisil ODS-3V (250x 4,6 mm, dp $5 \mu \mathrm{m}), \mathrm{FM}$ : acetonitrila:tampão fosfato 37,5 mmol/L a pH 7, 40:60 v/v e acetonitrila:tampão fosfato 37,5 mmol/L a pH 3, 35:65 v/v; vazão: $1 \mathrm{~mL} / \mathrm{min}$; volume de injeção: $2 \mu \mathrm{L}$; detecção: $U V$ a $254 \mathrm{~nm}$. Adaptado da ref. 31

grafitizado, ${ }^{32-35}$ devido à pequena difusão dos solutos nos poros destes suportes. Nestes casos, o aumento da temperatura diminui o fator de assimetria dos solutos neutros. Na Figura 3a o tempo de análise é de 70 min e os solutos 11 a 16 são fortemente retidos, apresentando fatores de assimetria elevados. Na Figura $3 \mathrm{~b}$ o aumento da temperatura ocasionou a redução dos fatores de assimetria, devido à maior difusão dos solutos na FE. ${ }^{32} \mathrm{O}$ aumento na difusão dos solutos com o aumento da temperatura é interessante nestas FE, pois a baixa eficiência obtida com estas é atribuída à pequena difusão dos solutos em seus poros. ${ }^{33}$

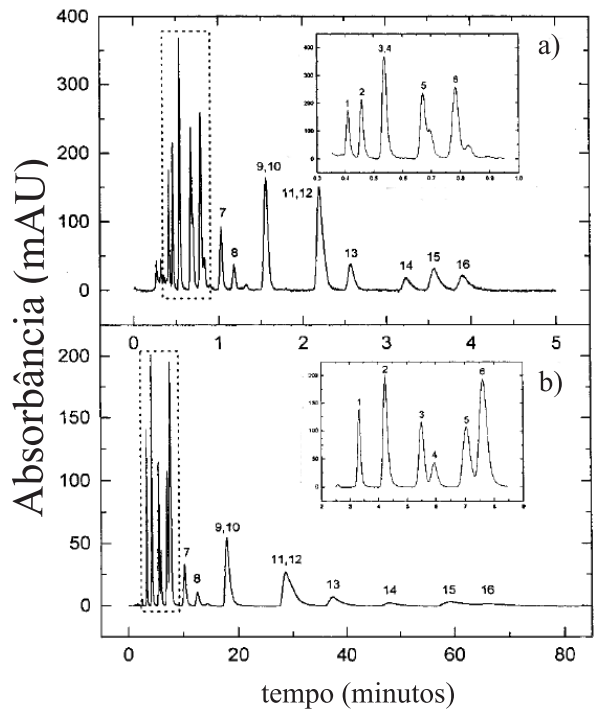

Figura 3. Separação de hidrocarbonetos poliaromáticos. Coluna: ZirChromPDB (100x 4,6 mm, dp 2,5 بm); FM: acetonitrila:água, 50:50 v/v; detecção: UV a $254 \mathrm{~nm}$. a) temperatura: $30{ }^{\circ} \mathrm{C}$; vazão: $1 \mathrm{~mL} / \mathrm{min}$; b) temperatura: 100 ${ }^{\circ} \mathrm{C}$; vazão: $5 \mathrm{~mL} / \mathrm{min}$. Identificação dos solutos: 1) naftaleno; 2) acenaftileno; 3) acenafteno; 4) fluoreno; 5) fenantreno; 6) antraceno; 7) fluoranteno; 8) pireno; 9) benzo[a]antraceno; 10) criseno; 11) benzo[b]fluoranteno; 12) benzo[k]fluoranteno; 13) benzo[a]pireno; 14) dibenzo[a,h]antraceno; 15) benzo[g,h,i]perileno; 16) indeno[1,2,3-cd]pireno. As expansões mostram a separação entre os solutos 1 a 6 . Adaptado da ref. 32

Adicionalmente as FE à base de óxido de zircônio possuem diversos sítios ácidos que interagem com solutos básicos, resultando em altos fatores de assimetria que podem ser reduzidos pelo aumento da temperatura, como demonstrado por Guillarme et al., ${ }^{3}$ onde o aumento da temperatura de 25 a $150{ }^{\circ} \mathrm{C}$ resultou na redução dos fatores de assimetria de derivados da cafeína, em uma análise conduzida na coluna Zirchrom-DB-C18 (50 x 4,6 mm, dp $3 \mu \mathrm{m})$.

\section{Influência da temperatura sobre a resolução}

A separação de hidrocarbonetos poliaromáticos ilustrada na Figura 3 a é um exemplo típico que poderia ser resolvido com um gradiente de FM, ${ }^{32}$ uma vez que a FM acetonitrila:água, 50:50 v/v, proporciona uma boa separação para os solutos de 1 a 6 , enquanto a retenção dos solutos 7 a 16 é excessiva. Por sua vez os solutos de 1 a 6 possuem pequenas entalpias de retenção e têm seus fatores de retenção pouco afetados pela temperatura, enquanto os solutos 7 a 16 possuem altas entalpias de retenção e têm seus fatores de retenção significativamente afetados pela temperatura, permitindo que o aumento da temperatura simule o efeito de um gradiente de FM, mantendo a separação dos solutos 1 a 6 (exceto pela coeluição dos solutos 3 e 4) e reduzindo o fator de retenção dos solutos 7 a 16 (Figura 3b).

Pereira et al..$^{34}$ demonstraram que gradientes de temperatura podem substituir gradientes de FM. Na Figura 4a, a separação é conduzida isotermicamente e com gradiente de FM. O soluto propanil, altamente apolar, não elui após 30 min de corrida, empregando-se a coluna Hypercarb, altamente retentiva para solutos apolares. A separação na Figura $4 \mathrm{~b}$ é conduzida utilizando FM isocrática e gradiente de temperatura, permitindo que todos os solutos sejam separados em $10 \mathrm{~min}$, mantendo uma resolução superior a 1,5. Adicionalmente, o aumento da vazão para $2 \mathrm{~mL} /$ min, possível devido à redução da viscosidade da FM, reduz o tempo de análise para 6 min e aumenta a resolução entre os picos devido à redução da difusão longitudinal. ${ }^{34}$

No exemplo da Figura 4a, com gradiente de FM, foram necessários 10 min para reequilibrar a coluna nas condições iniciais, enquanto no exemplo da Figura 4b, com gradiente de temperatura, os autores indicaram que a coluna foi reequilibrada nas condições inicias em apenas $3 \mathrm{~min}$, resultando em menores tempos de análise devido ao emprego de maiores vazões e da redução do tempo de reequilíbrio da coluna. ${ }^{34}$

No caso de misturas ainda mais complexas, eluição com gradientes de FM e temperatura podem ser utilizadas conjuntamente, para aumentar a seletividade, como no exemplo mostrado na Figura 5, onde a separação dos 20 solutos não foi alcançada isotermicamente a 50,70 ou $90{ }^{\circ} \mathrm{C}$ utilizando-se um gradiente de FM. Entretanto, o uso em conjunto de um gradiente de FM e de temperatura possibilitou a separação dos 20 solutos. ${ }^{36}$

\section{FASES MÓVEIS}

As FM utilizadas em HTLC são as mesmas utilizadas em HPLC e, como o aumento da temperatura diminui a viscosidade e a constante dielétrica da FM, é possível utilizar modificadores orgânicos viscosos e/ou fracos quando usados em modo reverso de eluição a temperatura ambiente.

\section{Água super aquecida como fase móvel}

A constante dielétrica $(\varepsilon)$ da água diminui de 80 , em temperatura ambiente, para valores de até 20 a $300{ }^{\circ} \mathrm{C} .{ }^{37-39}$ Assim, o uso da água como FM em altas temperaturas pode simular as propriedades de uma mistura da água com modificadores orgânicos. $\mathrm{O}$ uso de FM $100 \%$ aquosa é um exemplo de química verde, livre do alto custo na aquisição e no descarte de solventes orgânicos, e possibilita a detecção no UV em baixos comprimentos de onda ou acoplamento com o detector por ionização em chama (Flame Ionization Detector, FID). ${ }^{37-40}$

A seletividade em cromatografia empregando água super aquecida como fase móvel (Super Heated Water Chromatography, SHWC) é 

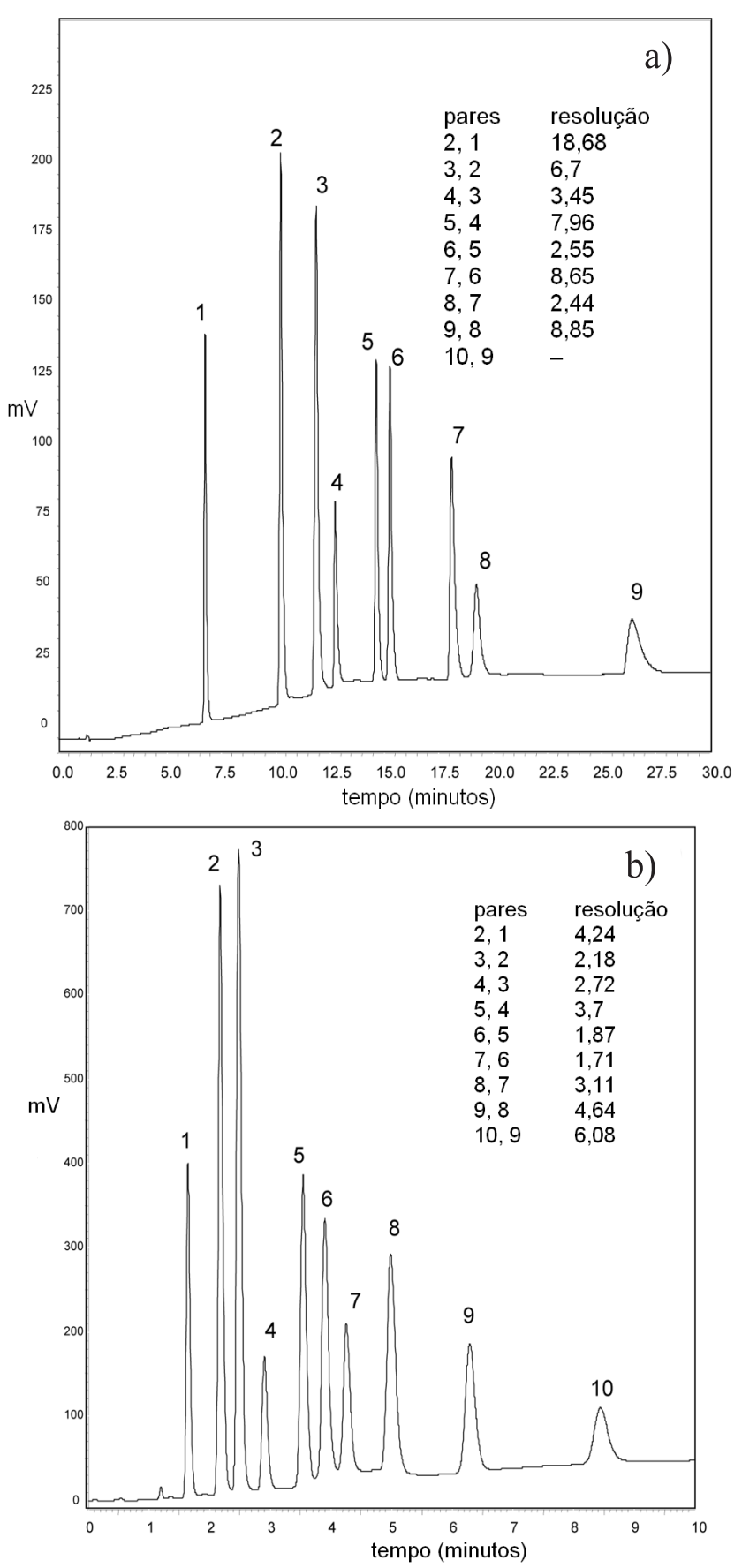

Figura 4. Separação de alguns agrotóxicos e metabólitos da atrazina. Coluna: Hypercarb (100x 4,6 mm, dp $5 \mu \mathrm{m})$. a) Temperatura: $40^{\circ} \mathrm{C}$; FM: gradiente de 5 a 100\% de acetonitrila em água em 15 min. b) Temperatura de 140 a 200 ${ }^{\circ} \mathrm{C}$ a $20{ }^{\circ} \mathrm{C} / \mathrm{min}, \mathrm{FM}$ : acetonitrila:água $50: 50 \mathrm{v} / \mathrm{v}$; vazão $1 \mathrm{~mL} / \mathrm{min}$. Identificação dos solutos: 1) atrazina-desetil-1-desisopropil; 2) atrazina-desetil; 3) atrazina-desisopropil; 4) propazina; 5) prometrim; 6) atrazina; 7) ametrim; 8) simazina; 9) simetrim; 10) propanil. Adaptado da ref. 34

controlada apenas pelo aumento da temperatura. A $190{ }^{\circ} \mathrm{C}$ a água é um eluente fraco em modo reverso de eluição, como relatado por Liu et al..${ }^{26}$ Estes autores obtiveram um tempo de retenção de 40 min para o pentilbenzeno em $\mathrm{FM} 100 \%$ água a $190{ }^{\circ} \mathrm{C}$ e de menos de $5 \mathrm{~min}$ em FM acetonitrila:água, $30: 70 \mathrm{v} / \mathrm{v}$, a $150^{\circ} \mathrm{C}$ na coluna XBridge $\mathrm{C} 18$ (150 x 4,6 mm, dp $5 \mu \mathrm{m}$ ) a uma vazão de $4 \mathrm{~mL} / \mathrm{min}$. Desta forma a técnica de SHWC é especialmente atrativa na análise de solutos polares que são fracamente retidos em modo reverso de eluição.

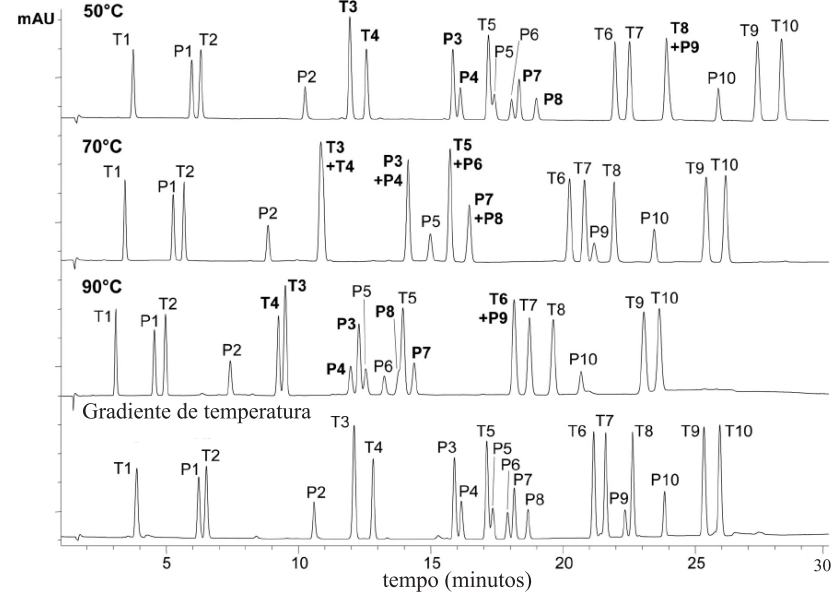

Figura 5. Análise de uma mistura de agrotóxicos. Coluna: Zorbax StableBondC18 (150 x 4,6 mm, dp 1,8 $\mu \mathrm{m})$; temperaturas: 50,70 e $90{ }^{\circ} \mathrm{C}$ e gradiente de temperatura: $40^{\circ} \mathrm{C}$ por $1 \mathrm{~min}, 40-60{ }^{\circ} \mathrm{C}$ a $1,3{ }^{\circ} \mathrm{C} / \mathrm{min}$ e $60-90{ }^{\circ} \mathrm{C}$ a $10^{\circ} \mathrm{C} / \mathrm{min}$. Em todas as corridas: FM: água:acetonitrila, $80: 20$ v/v a $45: 55$ v/v em $30 \mathrm{~min}$; vazão: $1 \mathrm{~mL} / \mathrm{min}$. Identificação dos solutos T1; desisopropilatrazina P1; fenurom T2; desetilatrazina P2; metoxurom T3; simazina, P3; metabenzitiazurom, T4; cianazina, P4; clorotolurom, T5; atrazina, P5; monolinurom, T6; sebutilazina, P6; diurom, T7; propazina, P7; isoproturom, T8; terbutilazina, P8; metobromurom, T9; prometrim, P9; linurom, T10; terbutrim, P10; cloroxurom. Adaptado da ref. 36

\section{Etanol como fase móvel}

As viscosidades da acetonitrila, do metanol e do etanol são de 0,4 , 0,6 e $1,2 \mathrm{cP} \mathrm{a} 25^{\circ} \mathrm{C}$, respectivamente. $\mathrm{O}$ uso da acetonitrila como modificador orgânico gera menor pressão e possibilita uma melhor resposta com detector UV a baixos comprimentos de onda. Por outro lado não há nenhuma restrição no uso de FM contendo álcoois quando a detecção é realizada a mais de $220 \mathrm{~nm}$ com o detector UV ou com um detector de massas. Além disso, a pressão do sistema pode ser aliviada pelo aumento da temperatura. ${ }^{41,42}$ Nosso grupo de pesquisa já realizou trabalhos com FM etanol:água onde o aumento da temperatura de 30 a $50{ }^{\circ} \mathrm{C}$ permitiu o uso de maiores vazões e a substituição das FM metanol:água e acetonitrila:água sem qualquer perda quanto a eficiência e a resolução. ${ }^{41}$

$\mathrm{O}$ uso de etanol como modificador orgânico, assim como a SHWC, é uma técnica limpa. O etanol é mais barato e menos tóxico que acetonitrila e metanol. O seu uso permite que a seletividade seja controlada por gradientes de solvente e/ou temperatura, uma vez que gradientes de temperatura de 30 a $150{ }^{\circ} \mathrm{C}$, na maioria dos casos, podem substituir gradientes de FM de 30 a $70 \%$ de acetonitrila. ${ }^{43}$.

Salvador et al. ${ }^{44}$ analisaram diversos cosméticos utilizando etanol como FM, obtendo limites de detecção (LD) de 0,5 a $2,7 \mu \mathrm{g} / \mathrm{mL}$. $\mathrm{O}$ aumento da temperatura de 20 a $45{ }^{\circ} \mathrm{C}$ ocasionou uma melhor seletividade entre os solutos, além do alívio da pressão do sistema, permitindo a análise simultânea de 18 filtros solares lipossolúveis como ilustrado na Figura 6. Vanhoenacker e Sandra ${ }^{36}$ utilizaram um gradiente de FM etanol:água para manipular a seletividade na separação de uma mistura de isômeros (Figura $1 \mathrm{~S}$, material suplementar).

Assim, devido à menor toxicidade e facilidades na aquisição e no descarte do etanol, FM com etanol pode ser utilizada em laboratórios que utilizam detecção no UV a mais de $220 \mathrm{~nm}$ ou detector de massas. As limitações quanto à pressão podem ser resolvidas utilizando temperaturas entre $40-60{ }^{\circ} \mathrm{C}$.

\section{FASES ESTACIONÁRIAS}

A sílica é o suporte ideal para cromatografia devido à homogeneidade de seus poros, alta área superficial e alta resistência mecânica. No 


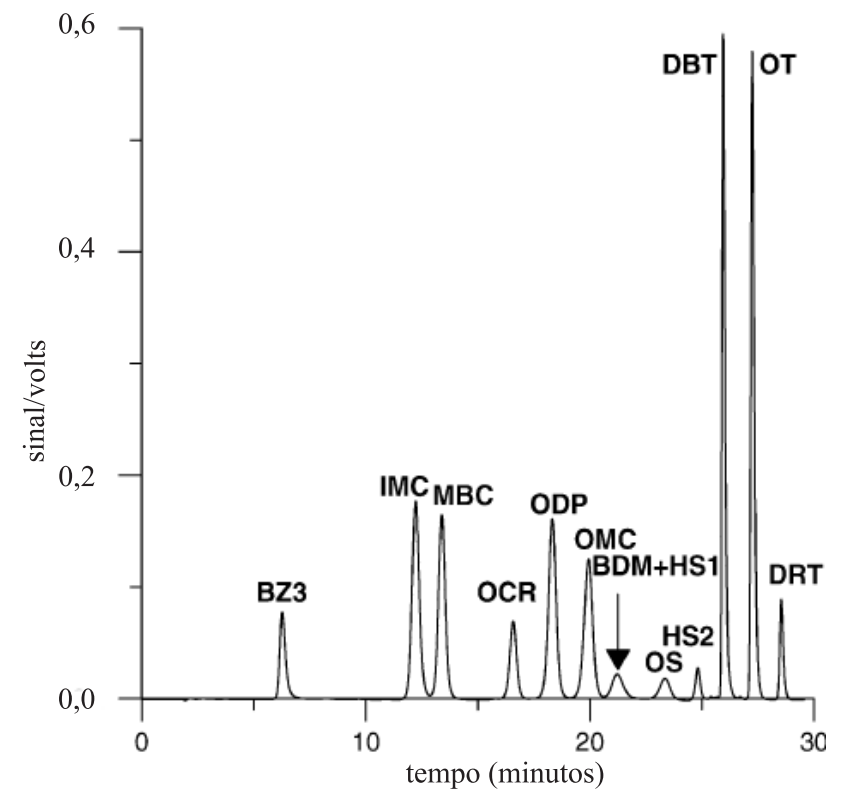

Figura 6. Separação de filtros solares lipossolúveis utilizando etanol como FM. Coluna: LiChrospher RP-18 $(125 \times 4 \mathrm{~mm}, \mathrm{dp} 5 \mu \mathrm{m})$. FM: etanol:ácido acético $1 \%, 60: 40$, v/v, por $5 \mathrm{~min}$, a $70 \%$ etanol em $10 \mathrm{~min}$, mantendo $70 \%$ etanol por $10 \mathrm{~min}$, a 100\% etanol em 0,5 min, mantendo $100 \%$ etanol por $4,5 \mathrm{~min}$, voltando a $60 \%$ em $14,5 \mathrm{~min}$; temperatura $45{ }^{\circ} \mathrm{C}$; vazão $0,5 \mathrm{~mL}$ min; injeção: $20 \mu L$; detecção: UV $313 \mathrm{~nm}$. Solutos: benzofenono-3 (BZ3), isoamilmetoxicinamato $(I M C)$, 4-metilbenzilidenocamfora $(M B C)$, octocrileno $(O C R)$, octildimetilPABA (ODP), octilmetoxicinamato $(O M C)$, butilmetoxidibenzoilmetano (BDM), homosalato (HS) onde HS1 e HS2 são isômeros de $H S$, octilsalicilato $(O S)$, dietil-hexilbutamidotriazina (DBT), octiltriazina (OT) e drometizoltrissiloxano (DRT). Adaptado da ref. 44

entanto, a sílica possui baixa estabilidade hidrolitica e se dissolve em meio alcalino, sendo que o aumento da temperatura acelera a dissolução da sílica. A maioria das FE à base de sílica se degrada rapidamente em FM contendo modificador orgânico:tampão fosfato a pH 7 em temperaturas entre 60 e $80{ }^{\circ} \mathrm{C}$. Por exemplo, a FE Zorbax Rx-C18 a $40{ }^{\circ} \mathrm{C}$ sofre uma dissolução mínima na FM acetonitrila:tampão fosfato $25 \mathrm{mmol} / \mathrm{L}$ a pH 7, 20:80 v/v, enquanto a $60^{\circ} \mathrm{C}$ a sílica do suporte é continuamente dissolvida. ${ }^{45}$ Até mesmo a FE de nova geração XTerra RP18 é totalmente degradada após a passagem de 6000 volumes de coluna de FM acetonitrila:tampão fosfato $20 \mathrm{mmol} / \mathrm{L}$ a pH 7, 30:70 v/v, a uma vazão de $1 \mathrm{~mL} / \mathrm{min}$ a $80{ }^{\circ} \mathrm{C} .{ }^{46}$

Devido à instabilidade hidrolítica da sílica, outros suportes foram utilizados como FE, como polímeros, carbono grafitizado e óxido de zircônio, suportes que se mostram estáveis em meio extremamente alcalino e descritos pelos seus fabricantes como úteis para HTLC, ${ }^{47}$ conforme mostra a Tabela 1. Entretanto, estes possuem baixas eficiências, poros irregulares e seletividades diferentes das FE à base de sílica, ${ }^{35,43}$ e não são ideais para HTLC uma vez que apresentam perda da FE (sangria), aumento da pressão e perdas de retenção e eficiência frente às reais condições de uso, onde a coluna é aquecida e resfriada várias vezes. ${ }^{43}$

As FE à base de óxido de zircônio são mais estáveis que as FE à base de sílica, mas se degradam rapidamente em FM contendo modificador orgânico:tampão fosfato a pH 7, entre 120 e $150{ }^{\circ} \mathrm{C}$. Nawrocki et $a l .{ }^{46}$ demonstraram que uma FE ZirChrom-PDB não sofre qualquer perda de eficiência após a passagem de 10.000 volumes de coluna de FM acetonitrila:tampão fosfato $20 \mathrm{mmol} / \mathrm{L}$ a pH 7, 30:70 v/v, a uma vazão de $1 \mathrm{~mL} / \mathrm{min}$ a $80^{\circ} \mathrm{C}$. Por outro lado Andersen et al..$^{48}$ demonstraram que a FE ZirChrom-PDB em FM acetonitrila:tampão fosfato $20 \mathrm{mmol} / \mathrm{L}$ a pH 7, 50:50 v/v, a uma vazão de $10 \mu \mathrm{L} / \mathrm{min}$ não sofre nenhuma perda de eficiência ou retenção após a passagem de 3.500 volumes de coluna a $100{ }^{\circ} \mathrm{C}$, enquanto a FE perde $80 \%$ de sua eficiência a $120^{\circ} \mathrm{C}$ e é totalmente degradada a $150^{\circ} \mathrm{C}$ após a passagem 1.500 volumes de coluna.

Problemas quanto à sangria das FE baseadas em óxido de zircônio foram observados para as FE ZirChrom DiamondBond, ZirChromCarb e ZirChrom-PBD por Marin et al. ${ }^{49}$ realizando gradientes de temperatura de 40 a $200{ }^{\circ} \mathrm{C}$ a uma taxa de $15^{\circ} \mathrm{C} / \mathrm{min}$, mantendo a $200{ }^{\circ} \mathrm{C}$ por $5 \mathrm{~min}$ em FM acetonitrila:água, 50:50 v/v.

Teutenberg et al. ${ }^{50}$ observaram a sangria das FE ZirChrom-Carb, Thermo Hypercarb e PLRP-S utilizando um detector de aerossol carregado (Charged Aerosol Detector, CAD) em FM 100\% água e gradientes de temperatura de 30 a $200{ }^{\circ} \mathrm{C}$ em $10 \mathrm{~min}$, mantendo a $200{ }^{\circ} \mathrm{C}$ por $10 \mathrm{~min}$.

Em um estudo posterior, Teutenberg et al..$^{51}$ estudaram as variações na pressão, retenção e eficiência das FE ZirChrom-Carb e Thermo Hypercarb a $185^{\circ} \mathrm{C}$ em FM $100 \%$ água a vazões de $1 \mathrm{~mL} /$ min e $0,5 \mathrm{~mL} / \mathrm{min}$, respectivamente. A cada $5 \mathrm{~h}$ de purga, as colunas eram resfriadas a $30{ }^{\circ} \mathrm{C}$ e reequilibradas em FM água:modificador orgânico, repetindo-se este procedimento por cinco vezes. Observouse que a FE ZirChrom-Carb apresentava, ao final do teste, uma pequena perda de retenção e um aumento de pressão (de 150 a 250 bar) enquanto a eficiência permanecia inalterada. Por sua vez, a FE Thermo Hypercarb não sofreu nenhuma alteração em sua retenção ou pressão, mas apresentou uma perda pronunciada de eficiência em virtude da alteração do leito cromatográfico, causada pela expansão e retração durante o processo de aquecimento e resfriamento.

Recentemente, Teutenberg et al. ${ }^{52}$ estudaram a estabilidade de algumas FE de nova geração descritas pelos fabricantes como úteis para HTLC (Tabela 1). As FE foram submetidas a $5 \mathrm{~h}$ de purga a 150 ${ }^{\circ} \mathrm{C}$ em FM metanol:água, 10:90 v/v, a uma vazão de $1 \mathrm{~mL} / \mathrm{min}$, resfriadas até $25^{\circ} \mathrm{C}$ e avaliadas com a mistura teste de Neue, ${ }^{53}$ repetindo-se este procedimento por cinco vezes. Neste estudo a maioria das FE à

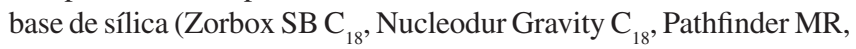
Ascentis $\mathrm{C}_{18}$, PackPro $\mathrm{C}_{16}$ ) e a híbrido Gemini $\mathrm{C}_{18}$ sofreram colapso durante o teste. As FE pHidelity $\mathrm{C}_{18}$ e Blaze $200 \mathrm{C}_{18}$ apresentaram uma variação no tempo de retenção de 4,1 a 3,0 min, um aumento da eficiência de 12.000 para 14.000 pratos e um decréscimo do fator de assimetria de 2,39 para 1,37 para o soluto teste naftaleno. A FE Gemini NX $\mathrm{C}_{18}$ apresentou uma pequena variação no tempo de retenção da amitriptilina e do propranolol, solutos básicos, e nenhuma alteração no tempo de retenção dos solutos neutros. As FE XBridge $\mathrm{C}_{18}$ e PLRP-S ficaram virtualmente inalteradas pelo teste, enquanto a FE ZirChrom PBD apresentou redução no tempo de retenção do acenafteno, de 10,2 a 9,2 min, e nenhuma alteração quanto à eficiência e assimetria. As FE que resistiram ao teste inicial com FM neutra foram submetidas a cinco ciclos com FM metanol:tampão fosfato $20 \mathrm{mmol} / \mathrm{L}$ a pH 2,2, 10:90 v/v, onde todas elas sofreram colapso, exceto a $\mathrm{FE}$ XBridge $\mathrm{C}_{18}$, que apresentou um pequeno aumento no fator de assimetria dos solutos teste após cinco repetições. Esta FE foi submetida a cinco ciclos de $150{ }^{\circ} \mathrm{C}$ em FM metanol:tampão fosfato $20 \mathrm{mmol} / \mathrm{L}$ a pH 12, 10:90 v/v, onde os solutos básicos começaram a apresentar fatores de assimetria significativos, mas ainda com resolução adequada.

Estes resultados indicam que as FE do tipo XBridge são mais estáveis que as demais FE. A estabilidade desta FE também foi atestada por Liu et al., ${ }^{26}$ que relataram uma perda de $10 \%$ na eficiência do butilbenzeno após 1 mês de uso a $200{ }^{\circ} \mathrm{C}$ com FM 100\% água.

\section{INSTABILIDADE TÉRMICA DOS SOLUTOS}

A instabilidade térmica dos solutos é um problema frequentemente superestimado em HTLC, uma vez que os solutos solvatados na 
Tabela 1. Colunas descritas pelos fabricantes como úteis para uso em temperaturas elevada

\begin{tabular}{|c|c|c|c|}
\hline Suporte & $\begin{array}{c}\text { Fase Estacionária } \\
\text { (Fabricante) }\end{array}$ & Tipos de FE disponíveis & T máx $\quad\left({ }^{\circ} \mathrm{C}\right)$ \\
\hline \multirow[t]{3}{*}{ Sílica com alta porcentagem de carbono e capeamento } & Acentis (Supelco) & $\mathrm{C} 18, \mathrm{C} 8$ & 60 \\
\hline & Nucleodur Gravity (Machery-Nagel) & C18, C8, HILIC & 60 \\
\hline & PackPro OS (YMC) & $\mathrm{C} 18$ & 80 \\
\hline Sílica protegida com grupos maiores & Zorbax StableBond (Agilent) & $\mathrm{C} 18, \mathrm{C} 8, \mathrm{C} 3, \mathrm{CN}$, Fenil & 90 \\
\hline \multirow[t]{3}{*}{ Sílica, polidentada } & Blaze 200 (Selerity) & $\mathrm{C} 18$ & 200 \\
\hline & pHidelity (Restek) & $\mathrm{C} 18$ & 200 \\
\hline & Cogent (MicroSolv) & C18, Colesterol & 100 \\
\hline Sílica híbrida orgânica-inorgânica com grupo metil & XTerra (Waters) & C18, C8, Fenil & 100 \\
\hline Sílica híbrida orgânica-inorgânica com grupo etil & XBridge (Waters) & $\mathrm{C} 18, \mathrm{C} 8$, Fenil & 200 \\
\hline Sílica, encapsulada com polímero & Pathfinder (Shimadzu) & $\mathrm{C} 18$ & 150 \\
\hline Sílica encapsulada com sílica híbrida com grupo metil & Gemini (Phenominex) & $\mathrm{C} 18$ & 60 \\
\hline Sílica encapsulada com sílica híbrida com grupo etil & Gemini NX (Phenominex) & $\mathrm{C} 18$ & 100 \\
\hline Sílica com interface orgânica-inorgânica & Eternity (Kromasil, AkzoNobel) & $\mathrm{C} 18$ & 80 \\
\hline \multirow[t]{2}{*}{ Óxido de zircônio } & Zirchrom (Zirchrom) & $\begin{array}{c}\text { PBD, PS, Carb, } \\
\text { Diamondbond-C18 }\end{array}$ & $\begin{array}{l}150 \\
200\end{array}$ \\
\hline & Discovery Zirconia (Supelco) & $\begin{array}{c}\text { PDB, PS } \\
\text { Carbon, Carbon C18 }\end{array}$ & $\begin{array}{l}150 \\
200\end{array}$ \\
\hline \multirow[t]{3}{*}{ Poliestireno/divinilbenzeno } & PLRP-S (Polymer Laboratories) & PS/DVB & 150 \\
\hline & PRP-1 (Hamilton) & PS/DVB & 150 \\
\hline & Jordi RP (Jordi Associates) & DVB, DVB- C18 & 150 \\
\hline Carbono grafitizado & Hypercarb (Thermo Scientific) & Carbono grafitizado & 200 \\
\hline \multirow[t]{2}{*}{ Óxido de zircônio } & Zirchrom (Zirchrom) & $\begin{array}{l}\text { PBD, PS, Carb, } \\
\text { Diamondbond-C18 }\end{array}$ & $\begin{array}{l}150 \\
200\end{array}$ \\
\hline & Discovery Zirconia (Supelco) & $\begin{array}{c}\text { PDB, PS } \\
\text { Carbon, Carbon C18 }\end{array}$ & $\begin{array}{l}150 \\
200\end{array}$ \\
\hline \multirow[t]{3}{*}{ Poliestireno/divinilbenzeno } & PLRP-S (Polymer Laboratories) & PS/DVB & 150 \\
\hline & PRP-1 (Hamilton) & PS/DVB & 150 \\
\hline & Jordi RP (Jordi Associates) & DVB, C18-DVB & 150 \\
\hline Carbono grafitizado & Hypercarb (Thermo Scientific) & Carbono grafitizado & 200 \\
\hline
\end{tabular}

FM possuem cinéticas de degradação menores do que no estado não solvatado, e o aumento da temperatura resulta na redução do tempo de análise, minimizando a degradação dos solutos. ${ }^{11,17,43,54}$

A degradação dos solutos depende do tipo de FE utilizada, onde sítios ácidos presentes na FE podem catalisar a degradação dos solutos. Giegold et al.$^{54}$ observaram como a FE utilizada pode catalisar a degradação dos solutos, estudando a variação na área do pico da talidomida em temperaturas entre 60 e $180^{\circ} \mathrm{C}$, em FM acetonitrila:ácido fórmico $0,1 \%, 20: 80 \mathrm{v} / \mathrm{v}$, a uma vazão de $0,2 \mathrm{~mL} / \mathrm{min}$ na coluna PLRP-S ( 150 x 4,6 mm, dp $3 \mu \mathrm{m})$ e em FM acetonitrila:ácido fórmico $0,1 \%, 25: 75 \mathrm{v} / \mathrm{v}$, a uma vazão de $1 \mathrm{~mL} / \mathrm{min}$ na coluna ZirChrom-Carb (150 x 4,6 mm, dp $3 \mu \mathrm{m})$, mantendo o mesmo tempo de retenção para a talidomida em ambas as colunas. Eles observaram que a área do pico da talidomida diminuiu na coluna ZirChrom-Carb quando a temperatura foi aumentada para valores acima de $60{ }^{\circ} \mathrm{C}$ e na temperatura de $180^{\circ} \mathrm{C}$ a talidomida foi totalmente degradada. Por outro lado, nenhuma variação na área do pico da talidomida foi observada na coluna PLRP-S quando a temperatura foi elevada de 60 a $180{ }^{\circ} \mathrm{C}$. (Figura 2S, material suplementar).

O menor tempo de análise ocasionado pelo aumento da temperatura (menores fatores de retenção e maiores vazões) aliado ao uso de uma FE com poucos sítios ácidos (Acquity BEH C18) permitiu que de Villiers et al. ${ }^{11}$ analisassem antocianinas termolábeis a $50{ }^{\circ} \mathrm{C}$.

\section{INSTRUMENTAÇÃO}

Os sistemas de HTLC são semelhantes aos sistemas convencionais de HPLC, exceto pela necessidade de pré-aquecer a FM na temperatura do forno para evitar gradientes indesejáveis de temperatura, utilizando uma serpentina de pré-aquecimento entre a coluna e o injetor, e de resfriar a FM antes que ela alcance o detector, quando este não é compatível com altas temperaturas, ${ }^{3,17,43,55}$ utilizando um tubo de resfriamento entre a coluna e o detector (Um esquema de um sistema para HTLC é descrito na Figura 3S, material suplementar).

O comprimento necessário da serpentina entre o injetor e a coluna é uma função da vazão e da temperatura empregada, ${ }^{3,55}$ sendo que quanto maior a vazão e a temperatura utilizada maior será o comprimento da serpentina. Por outro lado, quanto maior a serpentina maior será a dispersão e a pressão gerada no sistema, sendo que o mesmo raciocínio se aplica ao tubo de resfriamento. Desta forma, a falta de uma instrumentação específica para aquecer e resfriar a FM e a coluna sem alargamento dos picos era um fator limitante da técnica de HTLC. Atualmente, já se encontram fornos específicos para tal fim, comercializado pela Selerity Technologies ${ }^{56}$ e a ZirChrom Separations,${ }^{57}$ ambas capazes de pré-aquecer a FM com resistências e de resfriar a FM por convecção forçada, às custas de um aumento insignificante da dispersão e da pressão. ${ }^{55}$ As descrições comerciais destes encontram-se na Tabela 2. ${ }^{56,57}$

\section{Pré-aquecimento da FM}

Quando a temperatura da FM é menor que a temperatura do forno, a temperatura perto das paredes é maior do que no centro da coluna, gerando um gradiente de temperatura ao longo da coluna que distorce os picos e altera os fatores de retenção, resultando em baixas eficiências e repetibilidades. Quando maiores esta diferença 
Tabela 2. Especificações dos aquecedores comerciais para HTLC

\begin{tabular}{|c|c|c|}
\hline Característica & Polartherm 9000 & Metalox 200C \\
\hline Tamanho $(\mathrm{cm})$ & $51 \times 20 \times 41$ & $25 \times 15 \times 41$ \\
\hline Temperatura de trabalho & $\begin{array}{c}5{ }^{\circ} \mathrm{C} \text { abaixo da } \\
\text { temperatura ambiente } \\
\text { a } 200^{\circ} \mathrm{C}\end{array}$ & $\begin{array}{c}7{ }^{\circ} \mathrm{C} \text { abaixo da } \\
\text { temperatura ambiente } \\
\text { a } 200{ }^{\circ} \mathrm{C}\end{array}$ \\
\hline $\begin{array}{l}\text { Gradiente de tempera- } \\
\text { tura }\end{array}$ & $1-30^{\circ} \mathrm{C}$ & $\mathrm{N} / \mathrm{D} *$ \\
\hline $\begin{array}{l}\text { Aquecimento da FM } \\
\text { (volume) }\end{array}$ & $1 \mu \mathrm{L}$ & $10,5 \mu \mathrm{L}$ \\
\hline $\begin{array}{l}\text { Resfriamento da FM } \\
\text { (volume) }\end{array}$ & $\mathrm{N} / \mathrm{D}^{*}$ & $4,0 \mu \mathrm{L}$ \\
\hline $\begin{array}{l}\text { Taxa máxima } \\
\text { de aquecimento }\end{array}$ & $\mathrm{N} / \mathrm{D} *$ & $17,9 \mathrm{cal} / \mathrm{s}$ \\
\hline Vazão máxima & $\mathrm{N} / \mathrm{D} *$ & $\begin{array}{c}6 \mathrm{~mL} / \mathrm{min} \\
\text { com FM } 100 \% \text { água }\end{array}$ \\
\hline Fabricante & Selerity Technologies ${ }^{56}$ & ZirChrom Separations $^{57}$ \\
\hline
\end{tabular}

*N/D informação não disponível.

de temperatura e a vazão utilizada, maiores serão os efeitos causados pelo gradiente de temperatura gerado ao longo da coluna, sendo que os solutos mais retidos são mais afetados devido ao maior tempo de residência na coluna, ${ }^{2,3,17,43,55-60}$ como é descrito na Tabela 3 para a separação dos fármacos ilustrada na Figura 7.

Tabela 3. Efeito do pré-aquecimento da FM sobre o tempo de retenção e sobre a eficiência da salicilamina e do ácido salicílico nas separações ilustradas na Figura 7, conduzida em diferentes temperaturas e vazões. Adaptado da ref. 60

\begin{tabular}{lccccc}
\hline Condições & \multicolumn{4}{c}{ FM pré-aquecida } & \multicolumn{2}{c}{ FM não pré-aquecida } \\
\hline $\begin{array}{l}\text { Temperatura } \\
\left({ }^{\circ} \mathrm{C}\right)\end{array}$ & $\begin{array}{c}\text { Vazão } \\
(\mathrm{mL} / \mathrm{min})\end{array}$ & $\begin{array}{c}\text { Tempo de } \\
\text { retenção } \\
(\mathrm{min})\end{array}$ & $\begin{array}{c}\text { Pratos } \\
\times 10^{-3}\end{array}$ & $\begin{array}{c}\text { Tempo de } \\
\text { retenção } \\
(\mathrm{min})\end{array}$ & $\begin{array}{c}\text { Pratos } \\
\times 10^{-3}\end{array}$ \\
Salicilamida & & 3,1 & 13,0 & 3,21 & 13,2 \\
35 & 1 & 2,78 & 13,5 & 3,08 & 12,9 \\
50 & 1 & 2,62 & 13,5 & 2,89 & 12,7 \\
70 & 1 & 1,41 & 10,5 & 1,55 & 8,1 \\
70 & 2 & & & & \\
Ácido salicílico & & 4,74 & 12,0 & 5,01 & 12,1 \\
35 & 1 & 3,97 & 12,6 & 4,66 & 11,6 \\
50 & 1 & 3,54 & 12,3 & 4,19 & 11,0 \\
70 & 1 & 1,9 & 11,5 & 2,2 & 6,40 \\
70 & 2 & & & &
\end{tabular}

Colunas com diâmetros internos $>1 \mathrm{~mm}$ possuem altos calores específicos que dificultam o equilíbrio térmico entre a coluna, o forno e a FM. No entanto, como em sistemas comerciais a FM é préaquecida na temperatura do forno, os fabricantes atestam que estes tipos de colunas podem ser utilizadas com gradientes de temperatura e com alta repetibilidade. ${ }^{55-57}$ Teutenberg et al. ${ }^{61}$ realizaram a separação de diversos antibióticos, empregando gradientes de temperatura de 40 a $140{ }^{\circ} \mathrm{C}$ em uma coluna de 150 × 4,6 mm, resfriando a coluna a $40{ }^{\circ} \mathrm{C}$ em 4 min, obtendo uma boa repetibilidade nos tempos de retenção e nas áreas dos picos de 10 injeções consecutivas (Figura 4S, material suplementar).

No entanto, colunas capilares (diâmetros internos $\leq 1 \mathrm{~mm}$ ) possuem pequenos calores específicos e utilizam pequenas vazões, possibilitando um rápido equilíbrio térmico entre a coluna e a FM que entra na coluna, permitindo a utilização das mesmas sem pré-aquecer a FM. Greibrokk e Andersen ${ }^{62,63}$ apresentaram vários trabalhos com colunas capilares em faixas de temperatura entre 40 e $180^{\circ} \mathrm{C}$ sem pré-aquecer a FM e sem sofrer efeitos in-

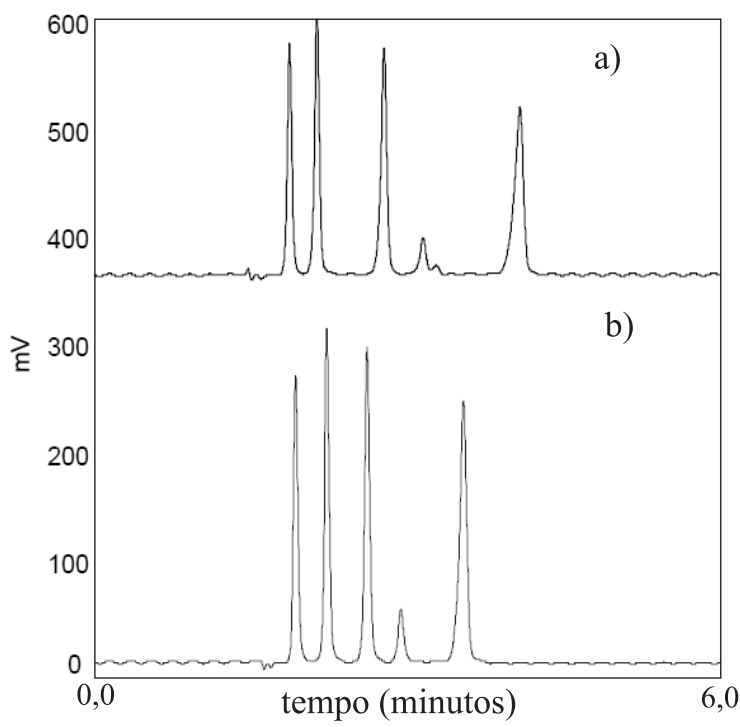

Figura 7. Separação de analgésicos a) sem o pré-aquecimento da FM e b) com o pré-aquecimento da FM. Coluna: Selerity Blaze C18 (100 x 4.6 mm, dp 3 $\mu \mathrm{m})$; temperatura: $70^{\circ} \mathrm{C} ; \mathrm{FM}$ : acetonitrila:ácido trifluoracético 0,1\%, 30:70 v/v; vazão: $2.0 \mathrm{~mL} / \mathrm{min}$; detecção: $U V 220 \mathrm{~nm}$. Ordem de eluição; acetominofeno, cafeína, salicilamida, aspirina e ácido salicílico. Adaptado da ref. 60

desejáveis. Partindo deste princípio, o grupo de pesquisa do Prof. F. M. Lanças da USP de São Carlos realiza um trabalho pioneiro no Brasil na área de HTLC com colunas capilares, utilizando gradientes de temperatura. ${ }^{64}$

\section{Resfriamento da FM}

O resfriamento da FM é importante apenas quando a temperatura utilizada agride o detector ou atrapalha na detecção. Como muitos detectores de UV não são compatíveis com temperaturas maiores que $80{ }^{\circ} \mathrm{C}$, o resfriamento da FM deve ser feito por meio de um tubo de resfriamento, colocado entre o fim da coluna e o detector, às custas de maiores dispersões e pressões. Os equipamentos comerciais possibilitam o resfriamento da FM antes que ela chegue ao detector, em detrimento de pequenos aumentos na dispersão e na pressão. ${ }^{43,55}$

\section{Detectores}

O efeito do aumento de temperatura pode ser benéfico ou maléfico na detecção dos solutos. Normalmente detectores como UV e fluorescência apresentam menor detectabilidade com o aumento da temperatura, enquanto detectores que operam em fase de vapor, como o detector de massas (mass spectrometry, MS) nos modos APCI-MS (atmospheric pressure chemical ionisation) e ESI-MS (electrospray ionization) e o detector ELSD (evaporative light scattering detector) apresentam maior detectabilidade com o aumento da temperatura. ${ }^{34,36,43}$

No entanto, qualquer afirmação quanto à melhoria da detecção em função do aumento da temperatura dever ser cautelosa. A resposta do detector de massas aumenta quando a composição da FM é mantida constante. ${ }^{36,43}$ Por outro lado, Pereira et al ${ }^{34}$ observaram que a altura do pico da sulfamerazina no modo de detecção APCI-MS aumentou quando a temperatura foi elevada de 30 a $180{ }^{\circ} \mathrm{C}$, mas o ruído também aumentou, fazendo com que a elevação da temperatura não proporcionasse nenhuma melhoria quanto à relação sinal/ruído. Já no modo ESI-MS a relação sinal/ruído aumentou quando a temperatura foi elevada de 30 a $90{ }^{\circ} \mathrm{C}$, mas diminuiu quando a temperatura foi aumentada de 90 a $180^{\circ} \mathrm{C}$. Desta forma, a influência da temperatura 
sobre a relação sinal/ruído depende do modo de detecção, do soluto e do instrumento empregado.

Por sua vez, o uso de FM 100\% aquosa permite que o detector tipo FID seja utilizado em HTLC, ${ }^{37-40}$ uma vez que a água não gera resposta neste detector. Entretanto, altas vazões de FM não são compatíveis com este detector. Yang et al. ${ }^{65}$ empregando as colunas Hypercarb $(10 \times 2,1$ $\mathrm{mm}$, dp $5 \mu \mathrm{m})$ e PRP-1 ( 25 x 4,1 mm, dp $5 \mu \mathrm{m})$ a vazões superiores a $1,24 \mathrm{~mL} / \mathrm{min}$, dividiram a vazão de FM que chegava ao detector, obtendo LD de 11-38 ng (injeção de 306-925 ng/ $\mu \mathrm{L}$ ) para divisões (split) de 1:10 e 1:17, respectivamente, para carboidratos e ácidos carboxílicos. Em um estudo posterior, Yang et al..$^{66}$ utilizaram uma coluna PRP-1 ( 250 x $0,5 \mathrm{~mm}$, dp $5 \mu \mathrm{m}$ ) e vazão de $20 \mu \mathrm{L} / \mathrm{min}$, direcionando toda a vazão para o detector, obtendo LD de 0,3-3 ng para aminoácidos.

\section{PERSPECTIVAS E LIMITAÇÕES}

O aumento da temperatura atenua as curvas de van Deemter e reduz a pressão dentro do sistema cromatográfico, permitindo que maiores vazões sejam utilizadas sem maiores penalidades quanto à pressão e à eficiência. A seletividade pode ser manipulada por gradientes de temperatura, substituindo ou sendo usados conjuntamente com gradientes de FM. Adicionalmente, a redução da viscosidade e da constante dielétrica da FM possibilita a redução nas quantidades de modificadores orgânicos utilizados, ou o uso de FM com etanol ou $100 \%$ água, fazendo da HTLC uma técnica limpa, rápida e barata.

Uma das maiores limitações da técnica de HTLC era a instabilidade das FE à base de sílica, enquanto as FE à base de outros suportes apresentavam baixas eficiências e seletividades distintas das FE à base de sílica. Atualmente as FE de nova geração, baseadas em sílica híbrida, como as FE do tipo XBridge, possuem as mesmas propriedades positivas que as demais FE à base de sílica e estabilidade superior a FE baseadas em outros suportes.

Desta forma, o desenvolvimento das FE do tipo Xbridge, aliado ao desenvolvimento de instrumentação especifica para HTLC, já disponível comercialmente, resultam em um grau de maturidade suficiente para que a HTLC se difunda para o meio industrial.

\section{AGRADECIMENTOS}

À FAPESP, ao CNPq e à CAPES pelo apoio financeiro e bolsas.

\section{REFERÊNCIAS}

1. Antia, F. D.; Horvath, Cs.; J. Chromatogr. 1988, 435, 1.

2. Yang, B.; Zhao, J.; Brown, J. S.; Blackwell, J.; Carr, P.W.; Anal. Chem. 2000, 72, 125.

3. Guillarme, D.; Heinisch, S.; Rocca, J. L.; J. Chromatogr., A 2004, 1052, 39.

4. Petersson, P.; Euerby, M. R.; J. Sep. Sci. 2007, 30, 2012

5. Sandra, P.; Vanhoenacker G.; J. Sep. Sci. 2007, 30, 241.

6. Edge, A. M.; Shillingford, S.; Smith, C.; Payne, R.; Wilson, I. D.; J. Chromatogr., A 2006, 1132, 206.

7. Xiang, Y.; Yan, B.; Yue, B.; McNeff , C. V.; Carr, P. W.; Lee M. L.; J. Chromatogr., A 2003, 983, 83.

8. Plumb, R.; Mazzeo, J. R.; Grumbach, E. S.; Rainville, P.; Jones, M.; Wheat, T.; Neue, U. D.; Smith, B.; Johnson, K. A.; J. Sep. Sci. 2007, 30, 1158.

9. Nguyen, D. T.-T.; Guillarme, D.; Heinisch, S.; Barrioulet, M. P.; Rocca, J. L.; Rudaz, S.; Veuthey, J. L.; J. Chromatogr., A 2007, 1167, 76.

10. Xiang, Y.; Liu, Y.; Lee, M. L.; J. Chromatogr., A 2006, 1104, 198.

11. de Villiers, A.; Cabooter, D.; Lynenc, F.; Desmet, G.; Sandra, P.; J. Chromatogr., A 2009, 1216, 3270.
12. Guillarme, D.; Grata, E.; Glauser, G.; Wolfender, J.-L.; Veuthey, J.-L.; Rudaz, S.; J. Chromatogr., A 2009, 1216, 3232.

13. Szabelski, P.; Cavazzini, A.; Kaczmarski, K.; Liu, X.; van Horn, J.; Guiochon, G.; J. Chromatogr., A 2002, 950, 41.

14. Goheen, S. C.; Gibbings, B. M.; J. Chromatogr., A 2000, 890, 73.

15. Hearn, M. T. W.; Zhao, G.; Anal. Chem. 1999, 71, 4874.

16. Heinisch, S.; Puy, G.; Barrioulet, M. P.; Rocca, J. L.; J. Chromatogr., A 2006, 1118, 234.

17. Heinisch, S.; Rocca, J.-L.; J. Chromatogr., A 2009, 1216, 642.

18. Castells, C. B.; Gagliardi, L. G.; Rafols, C.; Roses, M.; Bosch, E.; J. Chromatogr, A 2004, 1042, 23.

19. Castells, C. B.; Rafols, C.; Roses, M.; Bosch, E.; J. Chromatogr., A 2003, 1002, 41

20. Gagliardi, L. G.; Castells, C. B.; Rafols, C.; Roses, M.; Bosch, E.; J. Chromatogr, A 2005, 1077, 159.

21. Gagliardi, L. G.; Castells, C. B.; Ràfols, C. Rosés, M.; Bosch, E.; J. Sep. Sci. 2008, 31, 969.

22. Roses, M.; Subirats, X.; Bosch, E.; J. Chromatogr., A 2009, 1216, 1756.

23. Buckenmaier, S. M. C.; McCalley, D. V.; Euerby, M. R.; J. Chromatogr., A 2004, 1060, 117.

24. Cheng, Y.-F.; Walter,T. H.; Lu, Z.; Iraneta, P.; Alden, B. A.; Gendreau, C.; Neue, U. D.; Grassi, J. M.; Carmody, J. L.; O'Gara, J. E.; Fisk, R. P.; $L C G C$ N. Am. 2000, 18, 1162.

25. Al-Khateeb, L.; Smith, R. M.; J. Chromatogr., A 2008, 120, 61.

26. Liu, Y.; Grinberg, N.; Thompson, K. C.; Wenslow, R. M.; Neue, U. D.; Morrison, D.; Walter, T. H.; O'Gara, J. E.; Wyndham, K. D.; Anal. Chim. Acta 2005, 554, 144.

27. Shen; S.; Lee; H.; McCaffrey; J.; Yee; N.; Senanayake; C.; Grinberg; N.; Clark J.; J. Liq. Chromatogr. Relat. Technol. 2006, 29, 2823.

28. Coym, J. W.; Dorsey, J. G.; J. Chromatogr., A 2004, 1035, 23.

29. Al-Khateeb, L. A.; Smith, R. M.; Anal. Bioanal. Chem. 2009, 394, 1255.

30. Fornstedt, T.; Zhong, G.; Guiochon, G.; J. Chromatogr., A 1996, 741, 1.

31. McCalley, D. V.; J Chromatogr., A 2000, 902, 311.

32. Li, J.; Hu, Y.; Carr, P. W.; Anal. Chem. 1997, 69, 3884.

33. Ells, B.; Wang, Y.; Cantwell, F. F.; J. Chromatogr., A 1999, 835, 3.

34. Pereira, L.; Aspey, S.; Ritchie, H.; J. Sep. Sci. 2007, 30, 1115.

35. Smith, R. M.; Burgess, R. J.; Chienthavorn, O.; Stuttard, J. R.; $L C-G C$ Int. 1999, 12, 30; LCGC N. Am. 1999, 17, 938.

36. Vanhoenacker, G.; Sandra, P.; J. Sep. Sci. 2006, 29, 1822.

37. Hartonen, K.; Riekkola, M.-L.; Trends Anal. Chem. 2008, 27, 1.

38. Smith, R. M.; J. Chromatogr., A 2008, 1184, 441.

39. Yang, Y.; J. Sep. Sci. 2007, 30, 1131.

40. Guillarme, D.; Heinisch, S.; Gauvrit, J. Y.; Lanteri, P.; Rocca; J. L.; J. Chromatogr., A 2005, 1078, 22.

41. Ribeiro, R. L. V.; Botolli, C. B. G.; Collins, K. E.; Collins C. H.; J. Braz. Chem. Soc. 2004, 15, 300.

42. Welch, C. J.; Brkovic, T.; Schafer, W.; Gong, X.; Green Chem. 2009, 11, 1232 .

43. Teutenberg, T.; Anal. Chim. Acta 2009, 643, 1.

44. Salvador, A.; Chisvert, A.; Anal. Chim. Acta 2005, 537, 15.

45. Claessens, H. A.; van Straten, M. A.; Kirkland, J. J.; J. Chromatogr., A 1996, 782, 259

46. Nawrocki, J.; Dunlap, C.; Li, J.; Zhao, J.; McNeff, C. V.; McCormick, A.; Carr, P. W.; J. Chromatogr., A 2004, 1028, 1.

47. Vanhoenacker, G.; Sandra, P.; Anal. Bioanal. Chem. 2008, 390, 245.

48. Andersen, T.; Nguyen, Q.-N. T.; Trones, R.; Greibrok, T.; J. Chromatogr., A 2003, 1018, 7.

49. Marin, S. J.; Jones, B. A.; Felix, W. D.; Clark, J.; J. Chromatogr., A 2004, 1030, 255.

50. Teutenberg, T.; Tuerk, J.; Holzhauser, M.; Kiffmeyer, T. K.; J. Chromatogr., A 2006, 1119, 197.

51. Teutenberg, T.; Hollebekkers, K.; Wiese, S.; Boergers, A.; J. Sep. Sci. 2007, 30, 1101 
52. Teutenberg, T.; Hollebekkers, K.; Wiese, S.; Boergers, A.; J. Sep. Sci. 2009, 32, 1262.

53. Neue, U.; van Tran, K.; Iraneta, P. C.; Alden, B. A.; J. Sep. Sci. 2003, $26,174$.

54. Giegold, S.; Holzhauser, M.; Kiffmeyer,T.; Tuerk, J.; Teutenberg, T.; Rosenhagen, M.; Hennies, D.; Hoppe-Tichy, T.; Wenclawiak, B.; $J$. Pharm. Biomed. Anal. 2008, 46, 625.

55. McNeff, C. V.; Yan, B.; Stoll, D. R.; Henry, R. A.; J. Sep. Sci. 2007, 30, 1672.

56. http://www.selerity.com/main/Documents/Series9000Brochure.pdf, acessada em Março 2010.

57. http://www.zirchrom.com/pdf/2003ht.pdf, acessada em Março 2010.

58. Poppe, H.; Kraak, J. C.; J. Chromatogr. 1983, 282, 399.

59. Dolan, J. W.; J. Chromatogr., A 2002, 965,195.
60. http://www.selerity.com/main/Documents/EAS2005Preheater.pdf, acessada em Março 2010.

61. Teutenberg, T.; Goetze, H.-J.; Tuerk, J.; Ploeger, J.; Kiffmeyer, T. K.; Schmidt, K. G.; Kohorst, W. gr.; Rohe, T.; Jansen, H.-D.; Weber, H. J. Chromatogr., A 2006, 1114, 89.

62. Greibrokk, T.; Andersen, T.; J. Sep. Sci. 2001, 24, 899.

63. Greibrokk, T.; Andersen, T.; J. Chromatogr., A 2003, 1000, 743.

64. Lanças, F. M.; $34^{\text {th }}$ International Symposium on High Performance Liquid Phase Separations and Related Technique, Dresden, Alemanha, 2009.

65. Yang, Y., Jones, A. D., Mathis, J. A., Francis, M. A., J. Chromatogr., A 2002, 942, 231.

66. Yang, Y.; Kennedy, T.; Kondo, T.; J. Chromatogr. Sci. 2005, 43, 518. 


\section{POSSIBILIDADES E LIMITAÇÕES NO USO DA TEMPERATURA EM CROMATOGRAFIA LÍQUIDA DE FASE}

\section{REVERSA}

Endler Marcel Borges, Carla B. G. Bottoli e Carol.H. Collins*

Instituto de Química, Universidade Estadual de Campinas, CP 6154, 13083-970 Campinas - SP, Brasil

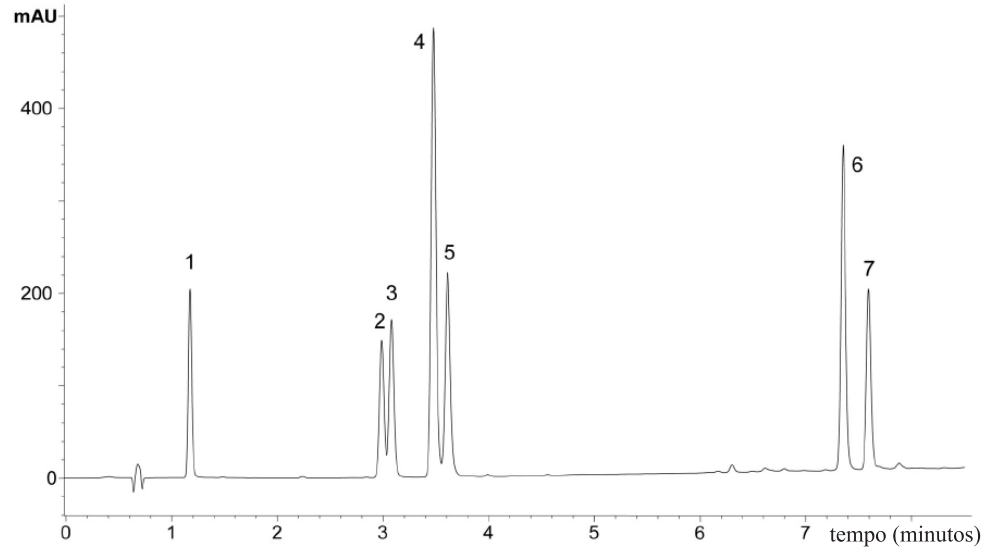

Figura 1S. Cromatografia verde de uma mistura de isômeros. Coluna: Zorbax StableBond-CN (150 x $3 \mathrm{~mm}$, dp 3,5 $\mu \mathrm{m})$; temperaura: $80^{\circ} \mathrm{C}$; FM: água:etanol 90: $10 \mathrm{v} / \mathrm{v}$ a 40:60 v/v em $10 \mathrm{~min}$; vazão: 1,2 mL/min; detecção: UV a $220 \mathrm{~nm}$. Identificação dos solutos: 1) anilina; 2) $\alpha$-naftilamina; 3) $\beta$-naftilamina; 4) $\alpha$-naftol; 5) $\beta$-naftol;

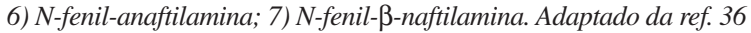
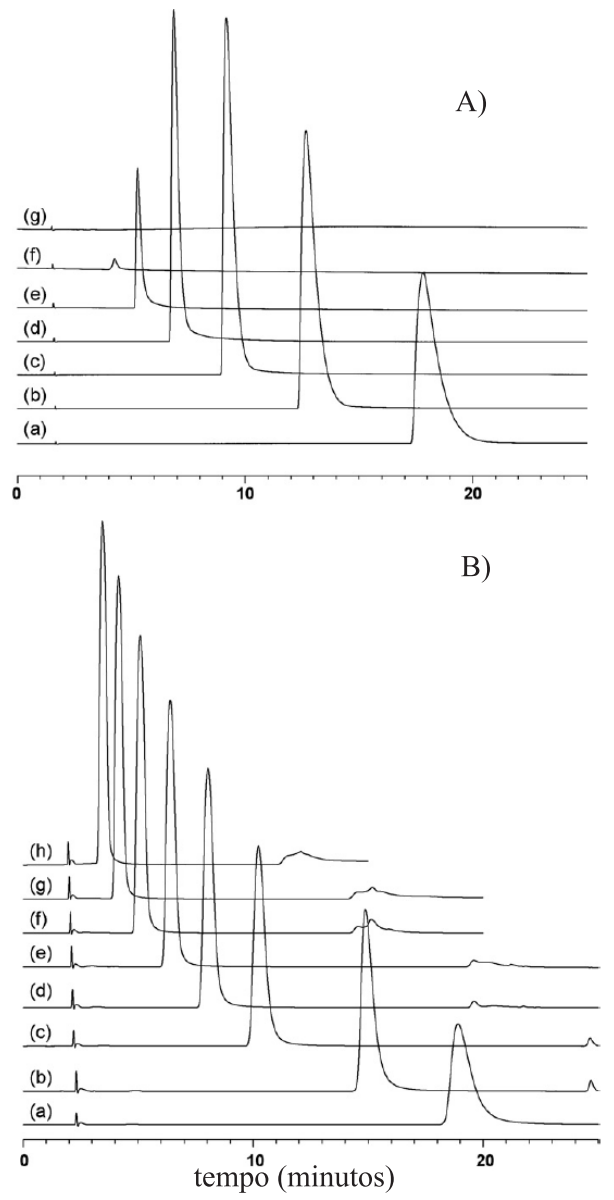

Figura 2S. Degradação da talidomida em duas colunas. A) Coluna: ZirChrom (150 mm $\times 4.6 \mathrm{~mm}, \mathrm{dp} 3 \mu \mathrm{m})\left(\right.$ a) $60^{\circ} \mathrm{Ca}(\mathrm{g}) 180^{\circ} \mathrm{C} \mathrm{com} \mathrm{incrementos} \mathrm{de} 20$

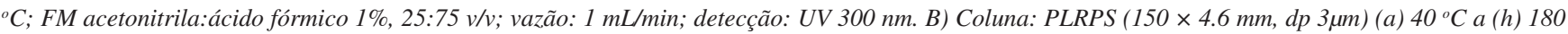
${ }^{\circ} \mathrm{C}$ com incrementos de $20^{\circ} \mathrm{C}$. FM acetonitrila:ácido fórmico $1 \%$, 20:80 v/v; vazão 0,2 mL/min; detecção: UV $300 \mathrm{~nm}$. Adaptado da ref. 54 


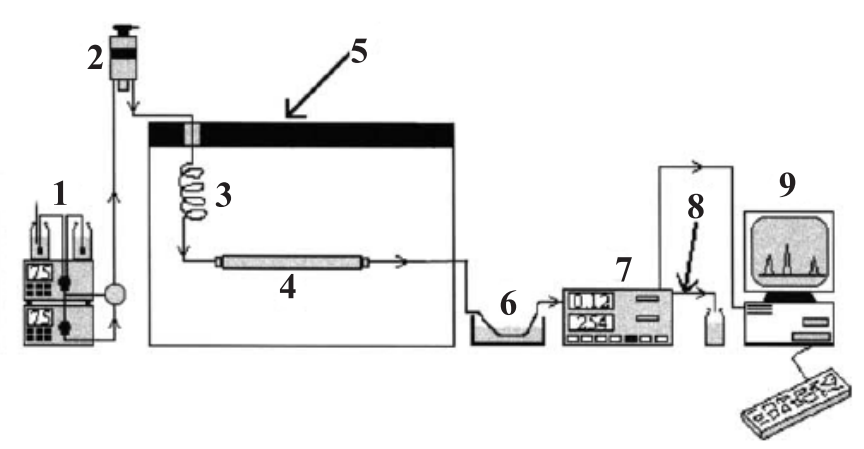

Figura 3S. Esquema de um sistema de HTLC: 1) bombas; 2) injetor; 3) serpentina de pré-aquecimento; 4) coluna; 5) forno; 6) tubo de resfriamento; 7) detector; 8) tubo para regular a pressão no detector; 9) sistema de aquisição de dados. Adaptado da ref. 3
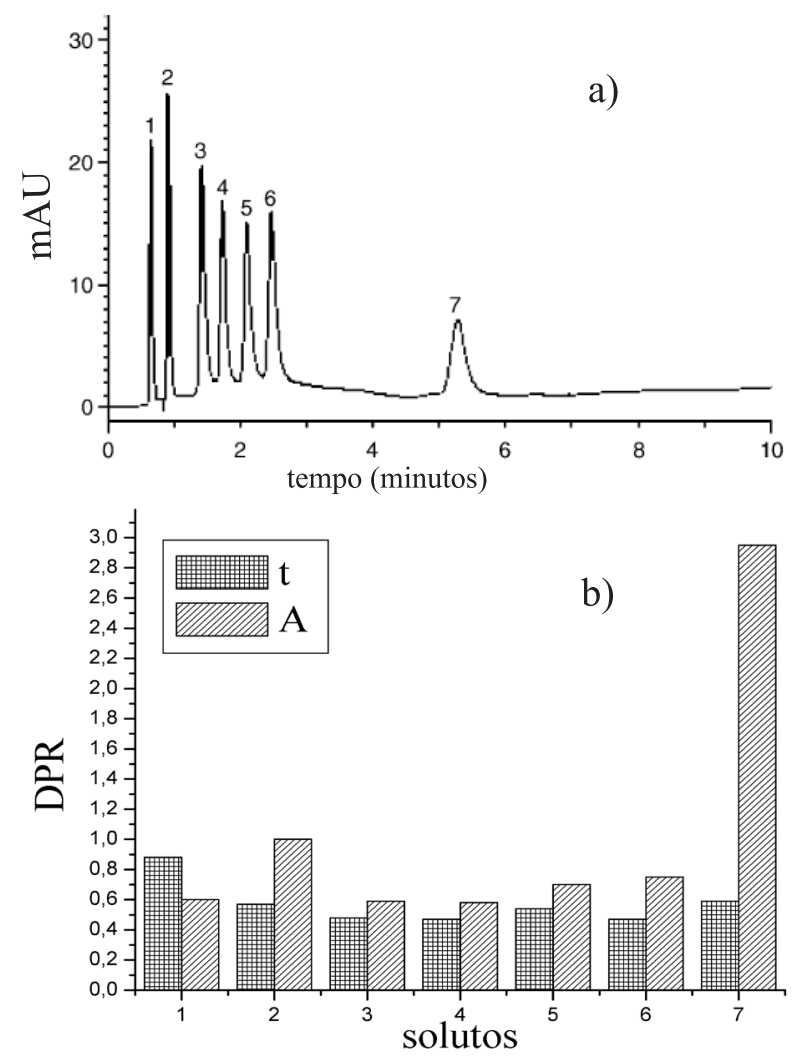

Figura 4S. Repetibilidade na separação de antibióticos. a) Separação em modo gradiente de temperatura de uma mistura de antibióticos. Coluna: ZirChromPS (4.6 ×150 mm, dp $3 \mu \mathrm{m})$. FM: ácido acético 0,1\%; temperatura: de 40 a $130^{\circ} \mathrm{C}$ em 4 min, mantendo a $130^{\circ} \mathrm{C}$ por $2 \mathrm{~min}$, de 130 a $40{ }^{\circ} \mathrm{C}$ em 4 min; vazão $2 \mathrm{~mL} / \mathrm{min}$; detecção: UV a $254 \mathrm{~nm}$. Identificação dos solutos: (1) citarabina, (2) 5-fluorouracila, (3) sulfadiazina, (4) sulfatiazol, (5) sulfametoxipiridazina, (6) clorampenicol, (7) etoposideo. b) Desvio padrão relativo (RSD) para 10 injeções consecutivas nos tempos de retenção (t) e nas áreas dos picos (A) dos solutos. Adaptado da ref. 61 